\title{
Verbesserter Betrieb von Simulated Moving Bed-Prozessen durch zyklische Modulation der Feedkonzentration*
}

Henning Schramm, Malte Kaspereit, Achim Kienle und

Andreas Seidel-Morgenstern *

\footnotetext{
* Der Artikel ist in englischer Sprache außerdem in Chemical Engineering \& Technology, 25 (2002) 12, S. 1151-1155 erschienen.

${ }^{* *}$ Dipl.-Ing. H. Schramm-Korrespondenzautor: schramm@mpi-magdeburg.mpg.de, Max-Planck-Institut Dynamik komplexer technischer Systeme, Sandtorstrasse 1, 39106 Magdeburg, Germany. Dipl.-Ing. M. Kaspereit: Max-Planck-Institut Magdeburg. Prof. Dr.-Ing. A. Kienle: Max-Planck-Institut Magdeburg, Otto-von-Guericke Universität Magdeburg, Universitätsplatz 2, 39106 Magdeburg, Germany.

Prof. Dr.-Ing. A. Seidel-Morgenstern: Max-Planck-Institut Magdeburg, Otto-von-Guericke Universität Magdeburg, Universitätsplatz 2, 39106 Magdeburg, Germany.
} 


\section{Kurzfassung}

Es wird eine neue Betriebsweise für chromatographische Simulated Moving Bed (SMB)-Prozesse vorgestellt. Während herkömmliche SMB-Prozesse mit konstanter Feedkonzentration betrieben werden, wird im Rahmen der vorliegenden Arbeit eine neue Betriebsweise mit zyklischer Modulation der Zulaufkonzentration vorgeschlagen. Hierdurch wird die Trennleistung im Vergleich zur herkömmlichen Betriebsweise signifikant verbessert. Alternativ zur Verbesserung der Produktreinheit kann bei gleichzeitiger Senkung des Lösemittelverbrauchs die Produktivität und die Konzentration der Zielkomponenten in den Produktströmen gesteigert werden.

\section{Einleitung}

In den letzten Jahrzehnten wurden chromatographische Trennprozesse zunehmend zur Isolierung und Aufreinigung von pharmazeutischen Wirkstoffen oder Feinchemikalien eingesetzt. Traditionell werden derartige Prozesse überwiegend diskontinuierlich durchgeführt. In neuerer Zeit setzen sich allerdings in zunehmendem Maße kontinuierliche Betriebsweisen durch.

Ein kontinuierlicher Betrieb wird mit Hilfe des Simulated Moving Bed-Verfahrens (Abb. 1) ermöglicht, das in den frühen sechziger Jahren von Universal Oil Products entwickelt wurde [1]. Hierbei wird die kontinuierliche Trennung einer binären Mischung durch einen simulierten Gegenstrom zwischen fester Adsorbensphase und flüssiger Lösemittelphase erreicht. Eine SMB-Apparatur besteht in der Regel aus vier Trennzonen, die durch zwei Zulaufstellen für Feed und Lösemittel und zwei Produktabzüge für Extrakt und Raffinat begrenzt werden. Die Bewegung der festen Adsorbensphase wird durch gleichzeitiges 
Neupositionieren (Schalten) dieser Zu- und Ablaufstellen in Richtung des Fluidstromes simuliert. Werden die Fluidströme und die Schaltzeit in geeigneter Weise gewählt, kann die stärker adsorbierte Komponente A kontinuierlich am Extraktausgang und die schwächer adsorbierte Komponente B kontinuierlich am Raffinat abgezogen werden.

\section{Abb. 1}

Für eine detailliertere Beschreibung des SMB-Prozesses sei an dieser Stelle auf die einschlägige Literatur verwiesen (z.B. [2, 3]).

Neben dem klassischen Konzept, bei dem der Prozess mit konstanten Betriebsparametern gefahren wird, sind in letzter Zeit neue Betriebsweisen entwickelt worden. Diese zeichnen sich durch eine Modulation der Betriebsparameter während der Schaltintervalle aus. Beim „VariCol“-Prozess [4] wird beispielsweise im Vergleich zum konventionellen Konzept eine Verbesserung der Trennleistung durch asynchrones Schalten der $\mathrm{Zu}$ - und Abläufe erreicht. Kerarney und Hieb [5] patentierten eine Betriebsweise bei der alle Fluidströme im Apparat innerhalb der Schaltintervalle variiert werden. Den gleichen Ansatz verfolgten auch Kloppenburg und Gilles [6], die eine signifikante Verbesserung eines industriellen Trennprozesses durch dynamische Optimierung der (variablen) Flussraten in jedem Schaltintervall erreichen konnten. Vor kurzem wurde außerdem eine neue Betriebsweise mit ausschließlicher Modulation des Feedstromes vorgeschlagen (Morbidelli und Mazzotti [7], Zang und Wankat [8]).

Im Gegensatz dazu wird in der vorliegenden Arbeit eine neue Betriebsweise vorgestellt, bei der innerhalb der Schaltintervalle die Konzentrationen der 
aufzutrennenden Komponenten im Feedstrom variiert werden („ModiCon“). Diese Methode kann mit bestehenden SMB-Anlagen in einfacher Weise durch die Verwendung von gebräuchlichen Gradientenpumpen realisiert werden. Die neue Betriebsweise zeichnet sich im Vergleich zu den anderen zyklischen Betriebsweisen insbesondere durch eine gleichbleibende Belastung und eine dadurch bedingte geringere Fehleranfälligkeit der Pumpen aus. Die Auslegung der Anlage ist vergleichsweise einfach und der Steuerungsaufwand für die Ventile ist gegenüber Betriebsweisen mit asynchronen Schaltstrategien geringer.

\section{Problemstellung}

Die Auswahl einer geeigneten Feedkonzentration ist eine wichtige Frage bei der Auslegung von SMB Prozessen. Es wurde bereits mehrfach gezeigt (z.B. [3, 9, 10]), dass die Produktivität eines SMB-Prozesses (d.h. die Produktmenge pro Zeit und Volumen an Adsorbensphase) mit steigender Feedkonzentration ansteigt. Der spezifische Lösemittelverbrauch, definiert als Lösemittelverbrauch im Verhältnis zur gewonnenen Menge an Wertkomponente, verringert sich gleichzeitig (Abb. 2).

Abb. 2

Die beiden Leistungskennzahlen Produktivität und Lösemittelverbrauch zeigen ein Sättigungsverhalten bei hohen Feedkonzentrationen. Der Verlauf der Kurven legt den Schluss nahe, dass jeweils die höchstmögliche Feedkonzentration ausgewählt werden sollte (begrenzt durch die Löslichkeit der Komponenten im Lösemittel), um die wirtschaftlichste Trennung zu erhalten. In vielen Fällen ist dieses Vorgehen jedoch nicht möglich. Die Robustheit, d.h. das Vermögen des Systems, in Anwesenheit von verschiedenen Störungen in 
einem bestimmten Betriebspunkt zu verharren, sinkt mit steigender Feedkonzentration. Höhere Konzentrationen erfordern typischerweise einen geringeren Feedstrom, welcher sich dann häufig nicht mit ausreichender Genauigkeit einstellen lässt. Bereits geringe Abweichungen der Flussraten, der Temperatur oder der Zulaufkonzentration können eine signifikante Verunreinigung der Produktströme zur Folge haben. Aus diesem Grund muss häufig eine geringere Zulaufkonzentration (deutlich unter der Löslichkeitsgrenze) gewählt werden. Hochkonzentrierte Feedgemische müssen oft verdünnt werden, um eine zufriedenstellende Trennung zu ermöglichen. Für diese Klasse von Prozessen bietet die neue Betriebsweise mit periodisch modulierter Feedkonzentration eine leistungsstarke Verbesserungsmöglichkeit im Vergleich zur konventionellen Betriebsweise. Durch eine Modulation der Feedkonzentration während der Schaltintervalle kann insbesondere die Produktivität deutlich gesteigert und gleichzeitig der spezifische Lösemittelverbrauch gesenkt werden.

\section{Neue SMB-Betriebsweise: Modulation der Feedkonzentration}

In der folgenden theoretischen Studie soll beispielhaft die Trennung zweier Cycloketone, Cyclopentanon (Komponente A) und Cycloheptanon (Komponente B), auf Silica Gel betrachtet werden. Alle für die Auslegung der Trennung verwendeten Parameter sind in Tab. 1 zusammengestellt. In der Simulation wird der SMB-Prozess durch das Gleichgewichts-Dispersionsmodell [11] beschrieben.

\section{Tab. 1}

Abbildung 3 zeigt die Konzentrationsverläufe im Inneren der SMB-Anlage in der Mitte eines Schaltintervalls für den zyklisch stationären Zustand. Die 
Konzentrationsprofile des SMB Prozesses mit herkömmlicher Betriebsweise (konstante Zulaufkonzentration) sind mit gestrichelten Linien markiert. Das Extrakt enthält nahezu reines Cyclopentanon (A). Cycloheptanon (B) kann am Raffinatausgang abgezogen werden. Durch entsprechende Positionierung der Konzentrationsfronten im Apparat wurden die konstanten Betriebsparameter des konventionellen SMB Prozesses hinsichtlich maximaler Produktivität und minimalem Lösemittelverbrauch optimiert [12].

\section{Abb. 3}

Für die neue Betriebsweise wird das Schaltintervall in zwei gleiche Abschnitte eingeteilt. Während der ersten Hälfte des Schaltintervalls wird anstelle der Feedmischung reines Lösemittel zugeführt, d.h. die Feedkonzentration ist null. In der zweiten Hälfte wird eine Feedmischung mit doppelter Konzentration im Vergleich zur herkömmlichen Betriebsweise verarbeitet. Da die SMB-Anlage mit jeweils gleichen Flussraten (Tab. 1) betrieben wird, ist damit in beiden Fällen der Feeddurchsatz gleich. Die entsprechenden Konzentrationsverläufe der Trennung mit variabler Feedkonzentration (abermals in der Mitte eines Schaltintervalls im zyklisch stationären Zustand) sind mit durchgezogenen Linien markiert (Abb. 3). Der Pfeil zeigt an, dass die Konzentrationsfront von Cyclopentanon (,langsame“ Komponente A) bei der neuen Betriebsweise in Trennzone III signifikant nach links verschoben ist. Hieraus folgt, dass die Raffinatreinheit im Vergleich zur konventionellen Betriebsweise wesentlich gesteigert wird. Im Gegensatz dazu wird die Reinheit des Produktstromes am Extrakt kaum beeinflusst.

Eine physikalische Interpretation des mit der neuen Betriebsweise verbundenen Effektes ist mit Hilfe der Gleichgewichtstheorie [13, 14] möglich. Hierbei wird 
davon ausgegangen, dass sich die Komponenten in Form von wandernden Fronten durch die verschiedenen Trennzonen bewegen und jede dieser Wellenfronten eine bestimmte Geschwindigkeit besitzt. Es treten zwei verschiedene Formen von Wellen auf: sogenannte Schockwellen und expansive Wellen. Bei den im betrachteten Beispiel vorliegenden LangmuirIsothermen treten Schockwellen in den Zonen III und IV und expansive Wellen in den Trennzonen I und II auf. Während sich jeder einzelne Punkt einer expansiven Welle mit unterschiedlicher Geschwindigkeit bewegt, ist die Geschwindigkeit einer Schockwelle für jeden Punkt der Welle gleich. Letztere Geschwindigkeit ist lediglich abhängig von der Höhe der Welle (d.h. von der Höhe des Schocks). Durch eine Beeinflussung der Plateau-Höhe der Konzentrationsfront der Komponente A in Trennzone III ist es somit möglich, die Geschwindigkeit dieser Front zu reduzieren und eine höhere Reinheit für die („schnelle“) Raffinat-Komponente B zu erzielen. Eine genauere Analyse dieses Effekts mit Hilfe der nichtlinearen Wellentheorie findet sich an anderer Stelle.

Durch die Modulation der Feedkonzentration konnte im Vergleich zum Referenzfall eine höhere Raffinat-Reinheit erzielt werden. Diese Reinheit liegt oberhalb des geforderten Wertes, der durch den optimierten konventionellen Prozess (Tab. 1) gerade erreicht wird. Alternativ können nun bei gleicher Produktreinheit die Flussraten für die neue Betriebsweise angepasst werden. Insbesondere kann die Konzentrationsfront von Cyclopentanon in Zone III durch eine Erhöhung des Feedstromes wieder in Richtung des Raffinatausgangs verschoben werden. Hieraus folgt - bei identischen Produktreinheiten - eine höhere Produktivität im Vergleich zur Betriebsweise mit konstanter Feedkonzentration. 
In Abb. 4 sind die Leistungskennzahlen beider Betriebsweisen - konstante (gestrichen) und variable (durchgezogene Linien) Feedkonzentration - in Abhängigkeit von der (gemittelten) Feedkonzentration dargestellt. Die Feedkonzentration der neuen Betriebsweise wurde in den beschriebenen zwei Schritten variiert - in der ersten Hälfte des Schaltintervalls wurde reines Lösungsmittel und in der zweiten Hälfte ein Gemisch mit doppelter Konzentration im Vergleich zur konventionellen Betriebsweise zugeführt. Beide Betriebsweisen wurden jeweils hinsichtlich minimalem Lösemittelverbrauch und maximaler Produktivität für die gewünschten Produktreinheiten von jeweils 95\% optimiert. Abb. 4a zeigt die entsprechende Abhängigkeit der Produktivität und Abb. 4b des spezifischen Lösemittelverbrauchs von der Feedkonzentration.

Abb. 4

Es ist offensichtlich, dass die Produktivität des SMB-Prozesses mit der neuen Betriebsweise signifikant höher ist als die des Prozesses mit konstanter Feedkonzentration. Gleichzeitig kann der spezifische Lösemittelverbrauch beträchtlich verringert werden.

Für die vorliegende Simulationsstudie wurden die Parameter einer realen Laboranlage verwendet. Die für diese Anlage geltenden Beschränkungen (Genauigkeit der Feedpumpe, gewünschte Produktreinheit) führen zu einer maximalen Feedkonzentration von 0,55 vol\% (Tab. 1) mit der mittels der konventionellen Betriebsweise die Trennung gerade noch durchgeführt werden kann. Bei höheren Konzentrationen ist der herkömmliche Betrieb der Anlage nicht mehr möglich, ohne die Reinheitsanforderungen zu verletzen. Bei der neuen Betriebsweise entspricht dieser Wert einer periodischen Modulation der 
Zulaufkonzentration zwischen 0 und 1,1 vol\%. Beide Betriebspunkte sind in Abb. 4 mit Pfeilen markiert. Im betrachteten Beispiel konnte die Produktivität durch eine einfache zweistufige Zugabe der Feedmenge um etwa $50 \%$ gesteigert werden, während der spezifische Lösemittelverbrauch gleichzeitig um $25 \%$ sank.

\section{Zusammenfassung}

In diesem Beitrag wurde eine neue Betriebsweise für SMB Prozesse vorgestellt. Durch eine zyklische Variation der Feedkonzentration (ModiCon) während des Schaltintervalls kann die Produktivität signifikant gesteigert und der spezifische Lösemittelverbrauch wesentlich verringert werden [15].

Das beschriebene Konzept ist nur für nichtlineare Prozesse anwendbar und wenn die Feedkonzentration im relevanten Bereich nicht durch die Löslichkeit der Komponenten limitiert ist. Für die Auslegung des neuen Prozesses ist die Kenntnis des Adsorptionsgleichgewichtes in höheren Konzentrationsbereichen notwendig. Die apparative Umsetzung an SMB-Anlagen ist durch herkömmliche HPLC-Gradientenpumpen oder einfache Ventilschaltungen problemlos möglich. Ein Vorteil gegenüber Betriebsweisen mit zyklisch variablen Fluidströmen ist die Tatsache, dass alle Pumpen in der Anlage mit gleichbleibender Belastung betrieben werden können und somit geringerem Verschleiß unterliegen.

Die Betriebsweise mit modulierten Feedkonzentrationen nutzt das nichtlineare thermodynamische Gleichgewicht zwischen fester und fluider Phase gezielt aus und ist deshalb nicht auf Trennungen mit Adsorptionsgleichgewichten vom Langmuir-Typ beschränkt. Für andere Adsorptionsgleichgewichte muss die 
Modulation der Feedzusammensetzung an das jeweilige dynamische Verhalten der Wellenfronten innerhalb der Anlage angepasst werden.

Abschließend sei darauf hingewiesen, dass die Aufteilung des Schaltintervalls in zwei Hälften nur zur Verdeutlichung der grundlegenden Effekte diente. Hierbei konnten bereits deutliche Verbesserungen erzielt werden. In Zukunft werden zusätzliche Untersuchungen zur Optimierung der zeitlichen Variation der Zulaufzusammensetzung durchgeführt. Hierbei sind unter anderem stufenweise Konzentrationsvariationen in mehr als zwei Schritten interessant. Außerdem soll das weitere Verbesserungspotential von SMB-Prozessen durch eine Kombination der neuen Betriebsweise mit anderen Konzepten (z.B. VariCol oder Modulation der Flussraten) untersucht werden. 


\section{Literatur}

[1] D.B. Broughton, C.G. Gerhold, US Patent 2985 589, 1961.

[2] D.M. Ruthven, C.B. Ching, Chemical Engineering Science, 1989, 44,1011-1038.

[3] M. Mazzotti, G. Storti, M. Morbidelli, Journal of Chromatography A, 1997, 769, 3-24.

[4] O. Ludemann-Hombourger, R.M. Nicoud, M. Bailly, Separation Science and Technology, 2000, 35, 1829-1862.

[5] M.M Kearney, K.L. Hieb, US Patent 5102 553, 1992.

[6] E. Kloppenburg, E.D. Gilles, Chemical Engineering and Technology, 1999, 22, 813-817.

[7] M. Morbidelli, M. Mazzotti, PREP, $15^{\text {th }}$ International Symposium on Preparative/Process Chromatography, Ion Exchange, Adsorption/Desorption Processes and Related Separation Techniques, Washington DC, USA, 16.-19.6. 2002, L-201, Book of Abstracts S. 53-54.

[8] Y. Zang, P.C. Wankat, Ind. Eng. Chem. Res., 2002, 41, 2504-2511.

[9] F. Charton, R.M. Nicoud, Journal of Chromatography A, 1995, 702, 97-112.

[10] G. Biressi, O. Ludemann-Hombourger, M. Mazzotti, R.M. Nicoud, M. Morbidelli, Journal of Chromatography A, 2000, 876, 3-15. 
[11] G. Guiochon, S. Shirazi, A. Katti, Fundamentals of Preparative and Nonlinear Chromatography, Academic Press, Boston, USA, 1994.

[12] H. Schramm, S. Grüner, A. Kienle, E.D. Gilles, In Proc. of the European Control Conference ECC'01, Porto, Portugal, 4.-7.9. 2001.

[13] H.K. Rhee, R. Aris, N.R. Amundson, First Order Partial Differential Equations, Prentice Hall, Englewood Cliffs, New Jersey 1989.

[14] F.G. Helfferich, P.W. Carr, Journal of Chromatography, 1993, 629, 97-122.

[15] H. Schramm, A. Kienle, M. Kaspereit, A. Seidel-Morgenstern, Patentanmeldung DE 10235 385.9, 2002. 


\section{Tabellen}

Tabelle 1: $\quad$ Modellparameter und Betriebspunkt für die Simulationsstudie.

\begin{tabular}{|c|c|c|}
\hline Parameter & Cyclopentanon (A) & Cycloheptanon (B) \\
\hline $\begin{array}{l}\text { Adsorptionsisothermen } \\
(\mathrm{q}, \mathrm{c}=\text { Konzentrationen in fester } \\
\text { bzw. flüssiger Phase) }\end{array}$ & $q_{A}=\frac{7.70 \cdot c_{A}}{1+0.148 \cdot c_{A}+0.110 \cdot c_{B}}$ & $q_{B}=\frac{5.72 \cdot c_{B}}{1+0.148 \cdot c_{A}+0.110 \cdot c_{B}}$ \\
\hline Anzahl Trennsäulen pro Zone & \multicolumn{2}{|c|}{$2 / 2 / 2 / 2$} \\
\hline Säulenlänge & \multicolumn{2}{|c|}{$25 \mathrm{~cm}$} \\
\hline Säulendurchmesser & \multicolumn{2}{|c|}{$2,0 \mathrm{~cm}$} \\
\hline Gesamtporosität & \multicolumn{2}{|c|}{0,83} \\
\hline Trennstufenzahl pro Säule & \multicolumn{2}{|c|}{100} \\
\hline Feedkonzentration & 0,55 vol\% & 0,55 vol\% \\
\hline Schaltintervall & \multicolumn{2}{|c|}{$3 \min$} \\
\hline Volumenstrom in Zone I & \multicolumn{2}{|c|}{$59,41 \mathrm{ml} / \mathrm{min}$} \\
\hline Extraktstrom & \multicolumn{2}{|c|}{$15,21 \mathrm{ml} / \mathrm{min}$} \\
\hline Raffinatstrom & \multicolumn{2}{|c|}{$8,83 \mathrm{ml} / \mathrm{min}$} \\
\hline Feedstrom & \multicolumn{2}{|c|}{$8,06 \mathrm{ml} / \mathrm{min}$} \\
\hline Vorgabe Produktreinheit & $95 \%$ (Extrakt) & 95\% (Raffinat) \\
\hline
\end{tabular}




\section{Abbildungen:}

Abb. 1:

Simulated Moving Bed-Prozess zur Trennung der Komponenten A und B.

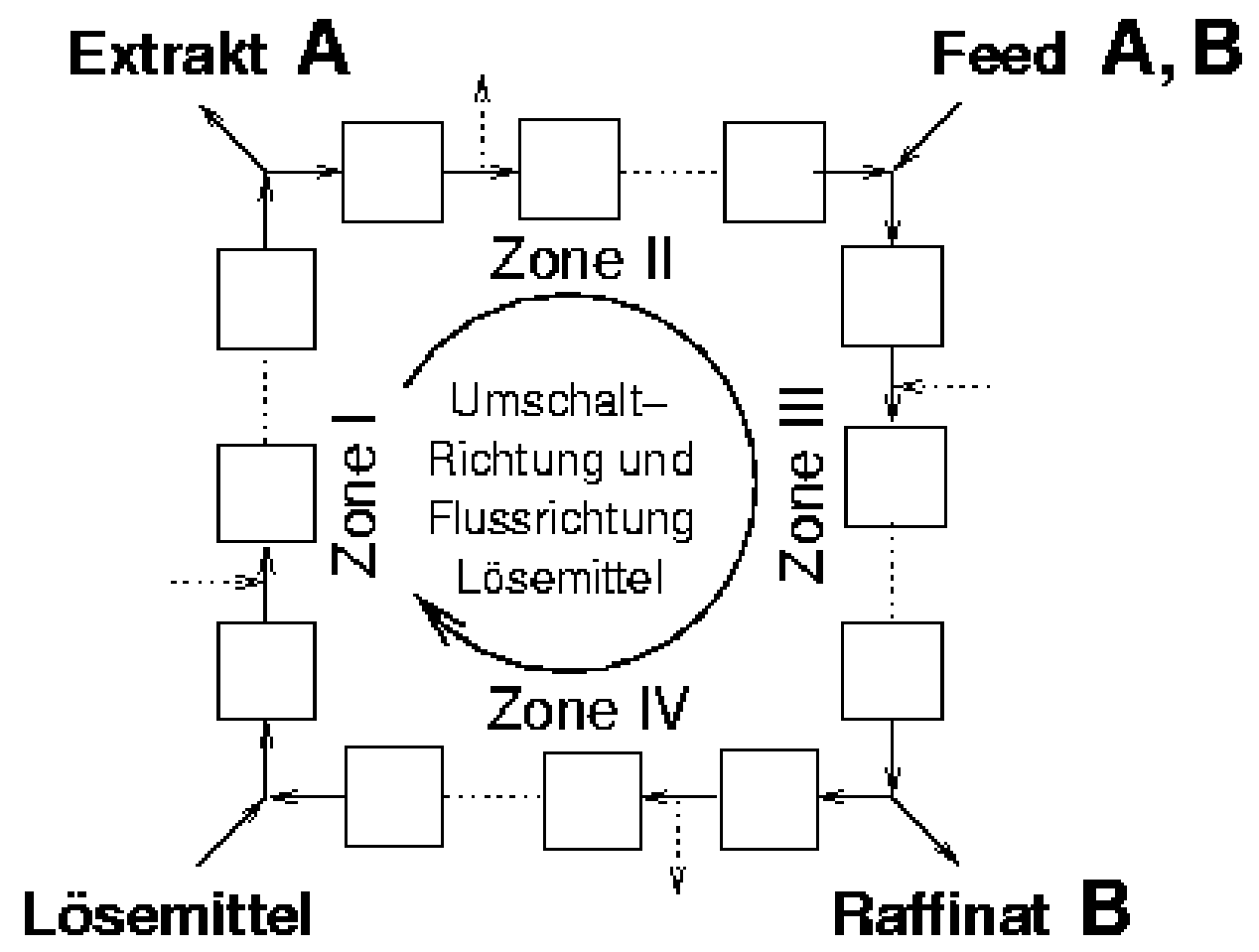


Abb. 2:

Qualitative Abhängigkeit der Produktivität, des spezifischen Lösemittelverbrauches und der Robustheit der Trennung von der gewählten Feedkonzentration bei einer vorgegebenen Produktreinheit (nach [3]).

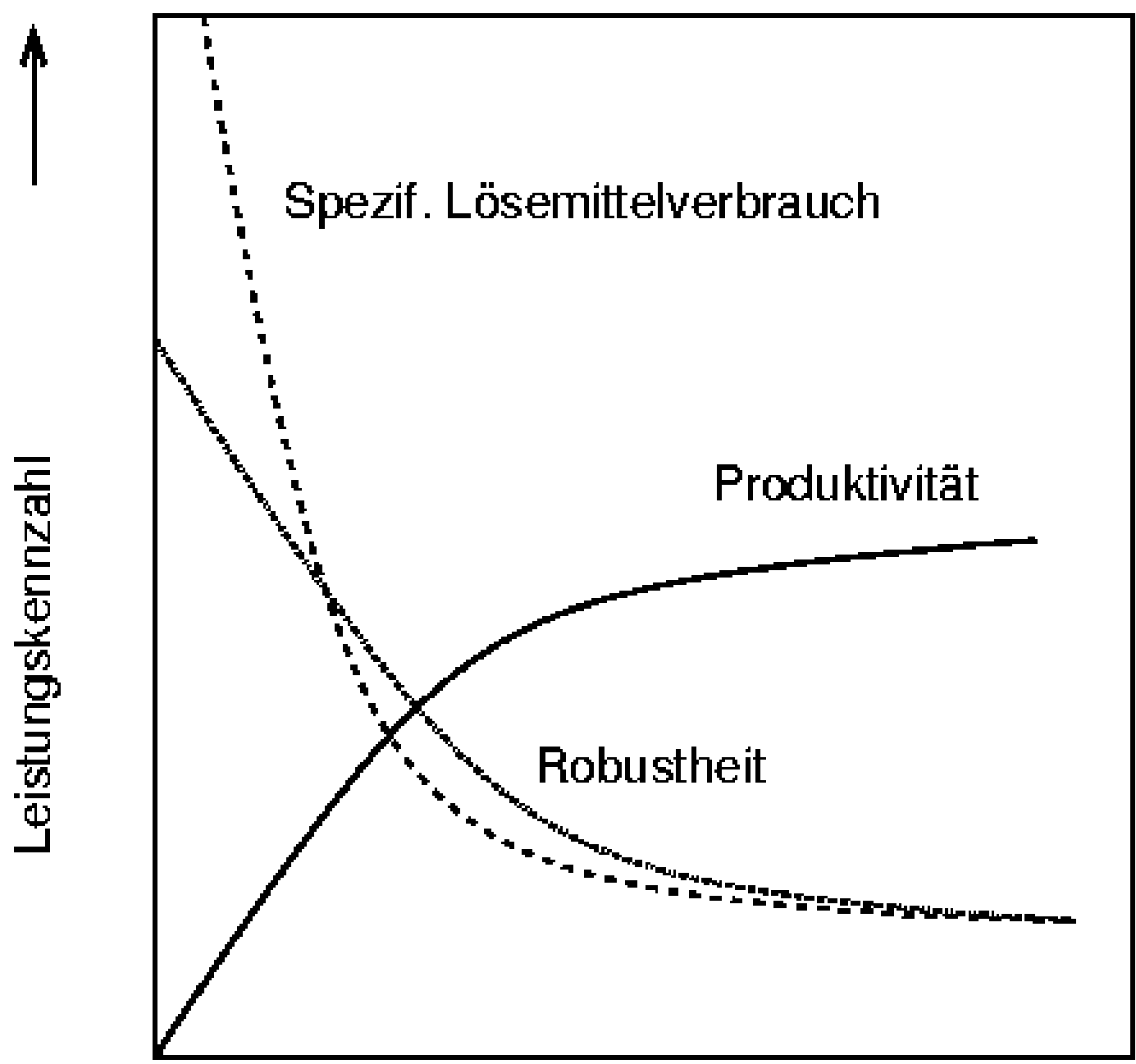

Feedkonzentration 
Abb. 3:

Konzentrationsverläufe für den herkömmlichen Prozess mit konstanten Parametern (gestrichelt) und der neuen Betriebsweise mit zyklischer Variation der Feedkonzentration (durchgezogen). Beide Prozesse werden mit identischen Flussraten betrieben und erreichen den gleichen Feeddurchsatz.

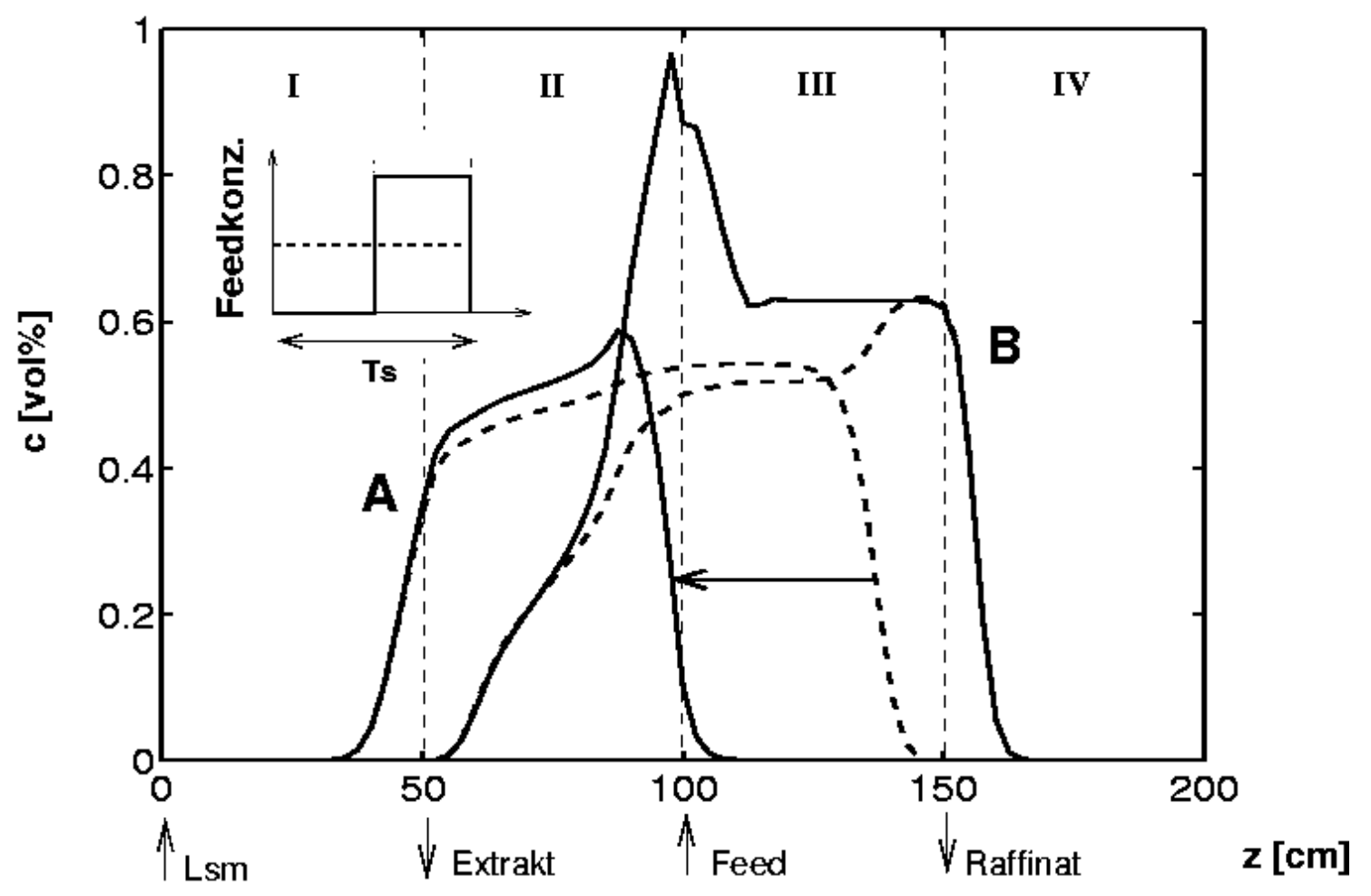


Abb. 4:

Leistungskennzahlen in Abhängigkeit von der Feedkonzentration für das betrachtete Beispiel. a) Produktivität, b) spezifischer Lösemittelverbrauch. Die Trennungen wurden jeweils hinsichtlich einer erforderlichen Produktreinheit von 95\% optimiert. Durchgezogene Linien: neue Betriebsweise, gestrichelt: herkömmlich.

a)

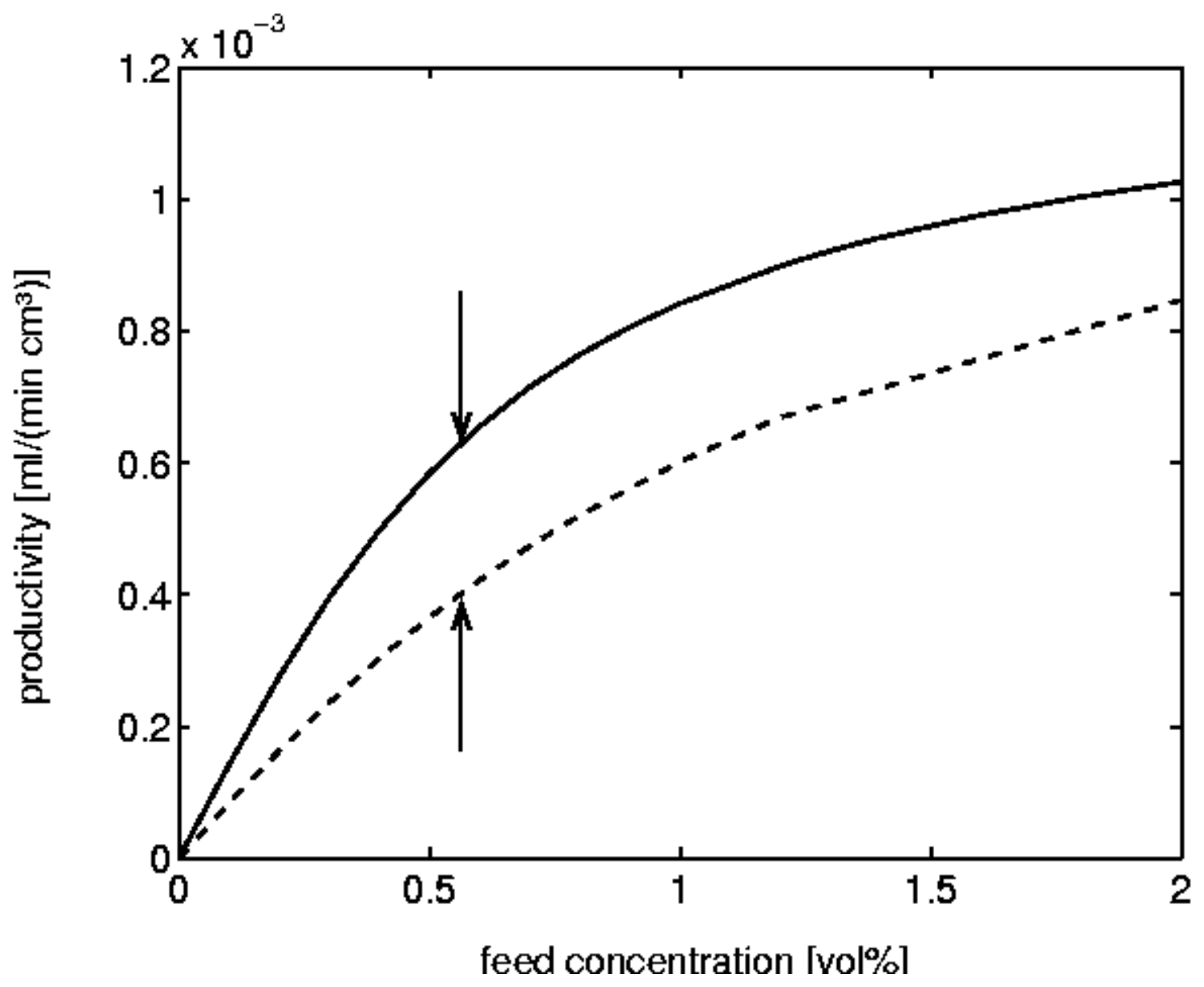


b)

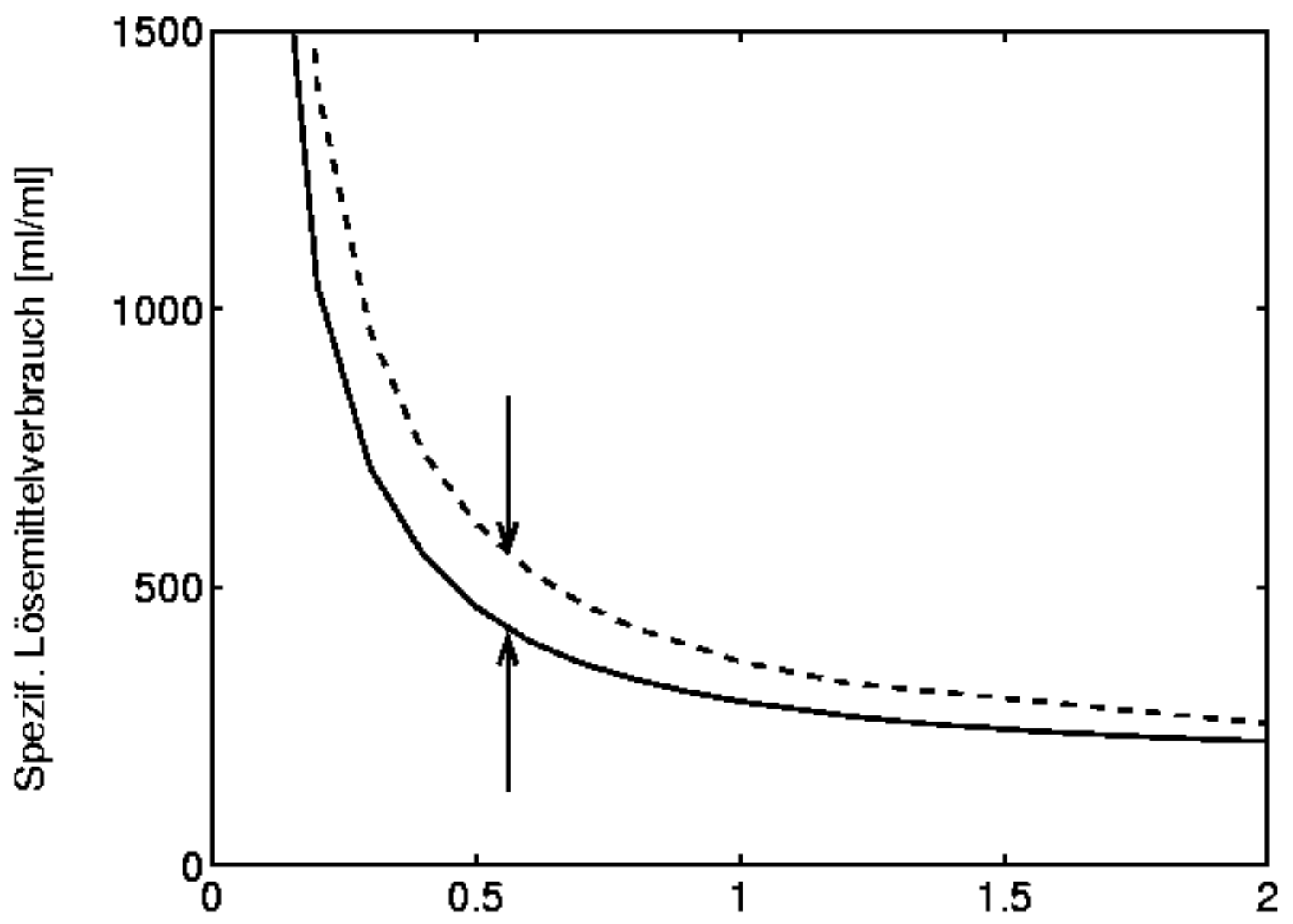

Feedkonzentration [vol\%] 